\title{
Dense estimation of layer motions in the atmosphere
}

\author{
Patrick Héas, Etienne Mémin, Nicolas Papadakis \\ IRISA, Université de Rennes 1, \\ 35042 Rennes Cedex, FRANCE \\ Patrick.Heas@irisa.fr, Etienne.Memin@irisa.fr, Nicolas.Papadakis@irisa.fr
}

\begin{abstract}
In this paper, we address the problem of estimating dense motion fields related to a stratified atmosphere which is observed through satellite imagery. Estimating the evolving vertical distribution of horizontal wind fields from satellite image time series is of great importance for the study of atmospheric dynamics. Because of the sparse 3-dimensional nature of observations, classical correlation-based techniques are not suited for the dense estimation of layer motion. Moreover, such methods are not necessarily temporally consistent. This paper proposes a sound energy-based estimator producing dense wind fields estimates for each partially observed atmospheric layer. The energy function to be minimized is composed of a data term based on the continuity equation which cancels out the influence of undesirable layers and of a specific div curl regularization term. To preserve the temporal consistency of the estimates, the variational method is initialized by propagation of the previous estimated field according to a velocity-vorticity formulation of Navier-Stokes equations. The relevance of our estimator is demonstrated on Meteosat image sequences.
\end{abstract}

\section{Introduction}

The analysis of complex fluid flows behaviors is a major scientific issue. In particular, understanding atmospheric dynamics is of great importance for meteorologists interested in weather forecasting, climate prediction, mesoscale system analysis, etc.

Introduction of radiosonde, automated balloon launchers, in-flight aircraft data have improved the estimation of wind fields and have been a subsequent step toward the understanding of meteorologic phenomena. However the network's temporal and spatial resolutions may be insufficient for the analysis of mesoscale dynamics. In an effort to avoid these limitations, geostationary satellite image sequences, characterized by higher acquisition rates and finer spatial resolutions, have been exploited by meteorologists.

Atmospheric motion estimators using satellite images have been developed since the seventies. Currently used methods for atmospheric motion estimation are based on cloud correlation in consecutive images. On one hand, these techniques constitute very fast methods and are generally locally very robust to noise. On the other hand, these approaches suffer from deficiencies (traceable features must be sufficiently contrasted and must persist over time on consecutive images), and therefore produce sparse results. The estimation is in addition local and thus prone to erroneous spatial variability, which can be reduced with the use of filters. To overcome these limitations, countour tracking techniques[4] or optical-flow methods dedicated to fluid motion have been recently introduced $[7 ; 2]$. These approaches have the main advantage to produce dense atmospheric motion fields.

Nevertheless, the underlying 3-dimensional nature of the projected scene may lead to erroneous results. Indeed, assuming that cloud layers are independent, they should not interfere during the estimation process. Furthermore, as the underlying associated optimization method are gradient descent techniques, a particular attention should be devoted to the initialization procedure.

We are interested in this work in deriving wind fields for specific cloud heights using satellite image sequences. The objective is to use the ability of a dense estimator dedicated to fluid flows while constraining the estimation to operate only on a specific atmospheric layer. This work also focus on initialization issues, by propagating in time a current estimate using a dynamic model.

To date no satisfying technique exists to extract accurately dense and temporally consistent wind fields related to different atmospheric layers. The remainder of this paper is organized as follows. Section 2 gives an overview on existing methods for the estimation of fluid flows. The proposed estimator dedicated to atmospheric layer motion is then explained in section 3, followed by some remarks on minimization issues. The propagation of motion fields between consecutive estimations using the vorticity equation is then detailed. Finally, the results described in section 4 demonstrate the interest of our approach.

\section{Related works on optical flow methods}

Standard estimator. Optical-flow estimation aims at recovering the apparent displacement field between two consecutive frames in an image sequence. Let $\mathbf{w}$ denote the un- 
known displacement field defined over the continuous plane domain $s \in \Omega$ and $f(s, t)$ the luminance function at point $s$ which is assumed to be continuous in space and time. The most accurate optical flow estimators used in computer vision are issued from Horn and Schunck method [6]. They are defined as the minimizer of an energy function $H=H_{1}+H_{2}$ composed of two terms. The first one assumes the constancy of the luminance of a point along its trajectory $\left(\frac{d f}{d t}=0\right)$. This so-called optical-flow constraint (OFC) is captured by letting :

$$
H_{1}(\mathbf{w}, f)=\int_{\Omega} \phi\left[\nabla f(s) \cdot \mathbf{w}(s)+\frac{\partial f(s)}{\partial t}\right] d s .
$$

The penalty function $\phi$ is usually the $L_{2}$ norm but it may be changed to a robust function attenuating the effect of data that deviate significantly from the OFC-based datamodel [1]. The second term $\mathrm{H}_{2}$ of the energy function is usually a standard first-order spatial smoothness term :

$$
H_{2}(\mathbf{w})=\alpha \int_{\Omega} \phi(\|\nabla \mathbf{w}\|) d s .
$$

where $\alpha>0$ is a parameter controlling the balance between the smoothness and the global adequacy to the brightness constancy assumption. Function $\phi$ may be the quadratic penalty if the searched solution is smooth everywhere or a robust norm function if one wants to handle implicitly the spatial discontinuities of the field [1].

Fluid dedicated estimator. The standard dense estimator defined as the minimizer of $H$ is generic.

First, in order to capture some specificities of image sequences with fluid motion, instead of the OFC-based datamodel, we may build the data term using the continuity equation :

$$
\frac{\partial \rho}{\partial t}+\operatorname{div}(\rho \mathbf{v})=0
$$

where $\rho$ denotes the fluid density, $\mathbf{v}=(u, v, w)^{T}$ is the $3 \mathrm{D}$ velocity and $d i v=\frac{\partial}{\partial x}+\frac{\partial}{\partial y}+\frac{\partial}{\partial z}$ stands for the divergence operator. Indeed, it has been demonstrated that the formation model of top of clouds infrared images is similar to the one used in transmittance imagery [3]. Thus, a vertical integration of the continuity equation (3) provides the constraint

$$
\frac{\partial f}{\partial t}+\operatorname{div}(f \mathbf{w})=0 .
$$

which can be introduced in the data term as

$$
H_{1}(\mathbf{w}, f)=\int_{\Omega} \phi\left[\operatorname{div}(f(s) \mathbf{w}(s))+\frac{\partial f(s)}{\partial t}\right] d s .
$$

However, such a data-model is highly sensitive to the presence of noise and is very likely not to hold everywhere. Also due to its differential nature, the model concerns velocity and not displacement. Therefore it is not valid in case of large displacements. Unlike the brightness constancy which can be directly expressed in a integrated form as $f(s+d(s), t+\Delta t)-f(s, t)=0$, the continuity equation, as it stands, cannot serve as the basis of an incremental data-model. To cope with this problem, velocities are assumed constant between the instants $t$ and $t+\Delta t$. In that case, equation (3) constitutes a simple first-order differential equation which can be integrated from $t$ to time $t+\Delta t$ along trajectories. Setting $\Delta t=1$ for notational convenience, the data-term $H_{1}(\mathbf{w}, f)$ is expressed as :

$$
\int_{\Omega} \phi[f(s+\mathbf{w}(s), t+1) \exp (\operatorname{div} \mathbf{w}(s))-f(s, t)] d s .
$$

Secondly, concerning the regularization term, it can be demonstrated that a first order regularization (Eq. 2) is not adapted to fluid phenomena as it favors the estimation of velocity fields with low divergence and vorticity. A second order regularization can advantageously be consider as proposed in [10] :

$$
H_{2}(\mathbf{w})=\alpha \int_{\Omega} \phi\left(\|\nabla \zeta(s)\|^{2}+\|\nabla \xi(s)\|^{2}\right) d s,
$$

where $\xi=c u r l \mathbf{w}=-\frac{\partial u}{\partial y}+\frac{\partial v}{\partial x}$ and $\zeta=d i v \mathbf{w}=\frac{\partial u}{\partial x}+\frac{\partial v}{\partial y}$ stand for the vorticity and the divergence of the 2D field $\mathbf{w}=(u, v)^{T}$. To circumvent the difficulty of implementing second order smoothness constraint, this regularization term can be simplified in a computational point of view in two interleaved first-order div-curl regularizations based on two auxiliary variables $\zeta_{1}$ and $\xi_{1}$ approximating the divergence and the vorticity of the flow [2]. Thus we have :

$$
\begin{array}{r}
H_{2}(\mathbf{w})=\alpha \int_{\Omega} \phi\left[\left(\zeta(s)-\zeta_{1}\right)^{2}+\beta\left\|\nabla \zeta_{1}\right\|^{2}\right. \\
\left.+\left(\xi(s)-\xi_{1}\right)^{2}+\beta\left\|\nabla \xi_{1}\right\|^{2}\right] d s,
\end{array}
$$

where $\beta$ is a positive regularization parameter. In order to avoid non-conditioned problems, a particular attention has been payed for the discretization of divergence and curl operator for which an uncentered discretization scheme has been used.

\section{Dense estimator for layer motion}

\subsection{Introduction of layers}

To construct a relevant dense estimator for layer motion, we propose to cancel out the influence of undesirable cloud layers by introducing a spatial mask in the data term of the energy function. Layers classifications based on cloud heights are produced automatically by a technique based on thresholds on the equivalent brightness temperatures [8]. These maps are routinely provided by the EUMETSAT consortium, the European agency which manages the Meteosat satellites. Let us denote by $C_{i}$ the class corresponding to the $i$-th layer and by $\mathbf{M}_{s \in C_{i}}$ the operator which is identity if $s \in C_{i}$ and which returns otherwise a fixed value out of the range taken by function $f(s, t)$. Employing a robust penalty function $\phi$, the new data term is written as

$$
\begin{array}{r}
H_{1}(\mathbf{w}, f)=\int_{\Omega} \phi[f(s+\mathbf{w}(s), t+1) \exp (\zeta(s)) \\
\left.-\mathbf{M}_{s \in C_{i}}(f(s, t))\right] d s .
\end{array}
$$


The first-order div-curl regularization term (Eq.8) is conserved. For both terms $H_{1}$ and $H_{2}$, M-estimator penalty function are chosen for their advantageous minimization properties [5]. The masking procedure together with the use of robust penalty function on the data term allows to discard implicitly the corresponding data from the estimation process. It is important to outline that the method always provides motion vector for all point $s$ of the image domain and areas corresponding to undesirable layers correspond to an interpolated wind field.

\subsection{Minimization issues}

In order to handle long range displacements, an incremental estimation of the dense displacement field is conducted through a multi resolution structure. Such process consists roughly in implementing estimation scheme on a pyramidal hierarchical representation of the image data associated to successive linearization around the estimate of coarser resolution. At a given resolution, a semi-quadratic energy function of the form $H=H_{1}+H_{2}$ is minimized. Two main sets of variables have to be estimated. The first one is the motion field $\mathbf{w}$, and the second one consists in the two auxiliary scalar fields $\xi_{1}$ and $\zeta_{1}$. The estimation is conducted alternatively by minimizing $H$ with respect to $\mathbf{w}, \xi_{1}$ and $\zeta_{1}$ respectively. For the motion field, considering the div and curl estimates $\xi_{1}$ and $\zeta_{1}$ as being fixed, the robust minimization with respect to $w$ is solved with an iteratively reweighted least squares technique. This optimization is embedded in an efficient multi-parametric adaptive multigrid framework. In turn, w being fixed, the minimization of $H$ with respect to $\xi_{1}$ and $\zeta_{1}$ is in fact equivalent to the minimization of $\mathrm{H}_{2}$ and is again conducted using an iteratively reweighted least square technique. More details of the minimization issues can be seen in [2].

\subsection{Vorticity propagation}

To perform a sound initialization, a motion field $\mathbf{w}$ estimated between time $t-1$ and $t$ is propagated up to time $t+1$ using a dynamic model.

The dynamic model for mesoscale winds in the lowest atmosphere part may be derived from Navier-Stokes equations. However, assuming sufficiently small horizontal density variations and neglecting vertical winds, we may avoid the pressure function estimation by employing its velocityvorticity form. For the horizontal wind field w, this model reads

$$
\frac{\partial \xi}{\partial t}+\mathbf{w} \cdot \nabla \xi+\xi \zeta=\frac{\mu}{\rho_{0}} \Delta \xi
$$

where $\rho_{0}$ is a known reference density function depending only on height. Divergence $\zeta$ is assumed to be a Gaussian random function with stationary increments. It thus defines a Brownian motion whose expectation asymptotically obeys to a heat equation of diffusion coefficient $\nu_{\zeta}$ :

$$
\frac{\partial \zeta}{\partial t}-\nu_{\zeta} \Delta \zeta=0
$$

The curl and divergence completely determine the underlying $2 \mathrm{D}$ velocity field and the current velocity estimate can be recovered from these quantities up to a laminar flow. Indeed, the Helmholtz decomposition of the field into a sum of gradient of two potential functions is expressed as

$$
\mathbf{w}=\nabla \times \Psi+\nabla \Phi+\mathbf{w}_{\text {lam }} .
$$

where $\mathbf{w}_{l a m}$ is the laminar part $\left(\right.$ div $\mathbf{w}_{l a m}=$ curl $\mathbf{w}_{l a m}=$ 0 ) of the field $\mathbf{w}$ and where the stream function $\Psi$ and the velocity potential $\Phi$ correspond to the solenoidal and the irrotationnal part of the field. The latter are linked to divergence and vorticity through two Poisson equations. Expressing the solution of both equations as a convolution product with the 2D Green kernel $G$ associated to the Laplacian operator : $\Psi=G * \xi, \quad \Phi=G * \zeta$, the whole velocity field can be recovered with the equation

$$
\mathbf{w}=\nabla \times(G * \xi)+\nabla(G * \zeta)+\mathbf{w}_{\text {lam }},
$$

which can be efficiently solved in the Fourier domain.

Thus, the vorticity and the divergence fields are developed from $t$ to $t+1$ using a discretized form of equations (10) and (11) and time increments $\Delta t$. After each time increment, assuming $\mathbf{w}_{\text {lam }}$ constant between the same time interval, equation (13) is used to update the velocity $\mathbf{w}$ needed by (10), with the current vorticity and divergence estimates. To avoid instability, an implicit time discretization scheme is used to discretize pde's (10) and (11). A classical centered finite difference scheme is used for the curl and divergence discretization. To solve the linear system associated to the implicit discretization scheme, the matrix has been constrained to to be diagonally dominant, which is a sufficient condition for a well-conditioned inversion problem. This condition reads $h \geq \max _{s}(|u|+|v|-|\zeta|)$, where the number of time increments $h=1 / \Delta t$.

\section{Experimental results}

Our experiments have been carried out on 2 Meteosat infrared images sequences of 15 frames acquired every 15 minutes. The first sequence exhibits a cyclone which appeared over the Indian ocean while the second sequence displays a perturbation over the North Atlantic ocean. In order to analyze visually both the temporal consistency and the accuracy of the estimation method, the trajectories of uniformly spread points have been reconstructed using a fourth-order Runge-Kutta integration method [9].

The visual inspection of Fig.1 outlines that our physically sound initialization procedure enhances temporal consistency of the wind fields estimates. Although the dynamics of the hurricane center are satisfactorily extracted with our without initialization, for accurate tracking of surrounding structures, wind fields need to be propagated in time. Indeed, several non-realistic trajectory discontinuities in the cyclone periphery disappear with our initialization scheme. Trajectories of the North Atlantic highest layer shows that 


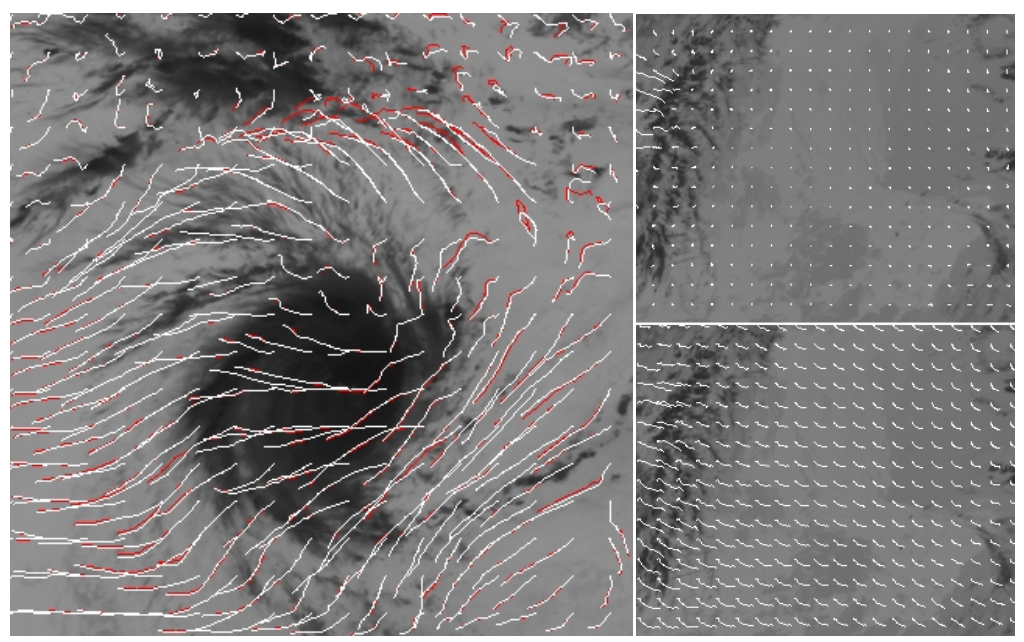

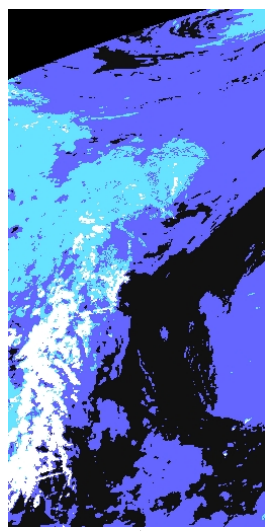

a)

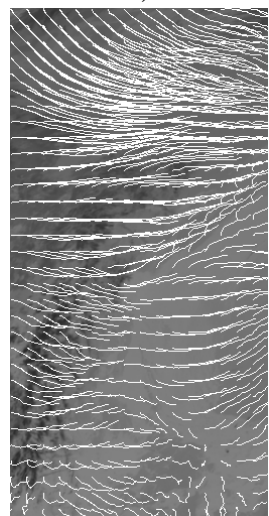

c)

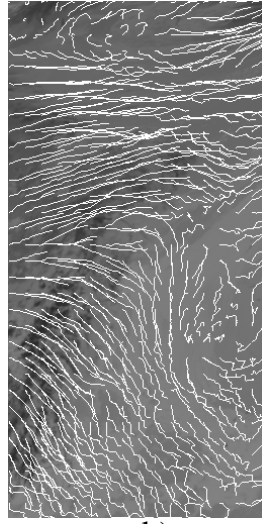

b)
Figure 1. Initialization contribution. Left: comparison between trajectories related to a cyclone estimated with (red plot) and without (white plot) our initialization scheme. Right: comparison between trajectories related to high layer dynamics estimated with (below) and without (above) our initialization scheme.

temporal consistency brought by wind field propagation may also enhance, thanks to the div-curl regularization, spatial homogeneity in the case of low amount of observations. The relevance of the layer dedicated estimation is demonstrated on the same North Atlantic area. Indeed, Fig.2 enables the visual comparison of trajectories corresponding to a layer mixture and trajectories corresponding to 3 different atmospheric layers. This layer decomposition reduces significantly the complexity of the 2D projected flow. Homogeneity of wind fields is enhanced and erroneous estimates near layer frontiers are removed. In particular, non-relevant average trajectories have been estimated in these areas for the layer mixture, while 2 perpendicular trajectories have been reconstructed for the middle and high cloud layers.

\section{Conclusions}

In this paper, we have presented a new method for the estimation of dense layer motion from satellite image sequences. The proposed estimation is a minimization-based approach where the observation term has been constrain to only consider specific layers. A physical sound initialization procedure has been introduced to preserve temporal consistency of the wind field estimates. Experimental results have demonstrated the interest of such an approach to analyze 3D atmospheric motions.

\section{References}

[1] M. Black and P. Anandan. The robust estimation of multiple motions: Parametric and piecewise-smooth flow fields. Computer Vision and Image Understanding, 63(1):75-104, 1996.

[2] T. Corpetti, E. Mémin, and P. Pérez. Dense estimation of fluid flows. IEEE Trans. Pattern Anal. Machine Intell., 24(3):365$380,2002$.
Figure 2. Layer dedicated estimation. Comparison between trajectories estimated for a layer mixture $(b)$ and trajectories estimated for the low (c), middle $(d)$ and high $(e)$ cloud layers according to classification maps such as (a).

[3] J. Fitzpatrick. The existence of geometrical density-image transformations corresponding to object motion. Comput. Vision, Graphics, Image Proc., 44(2):155-174, Nov. 1988.

[4] P. Gamba. Meteorological structures shape description and tracking by means of bi-rme matching. IEEE trans. on Geoscience and Remote sensing, 37(2):1151-1161, Mar. 1999.

[5] P. Holland and R. Welsch. Robust regression using iteratively reweighted least-squares. Commun. Statis.-Theor. Meth., A6(9):813-827, 1977.

[6] B. Horn and B. Schunck. Determining optical flow. Artificial Intelligence, 17:185-203, 1981.

[7] R. Larsen, K. Conradsen, and B. Ersboll. Estimation of dense image flow fields in fluids. IEEE trans. on Geoscience and Remote sensing, 36(1):256-264, Jan. 1998.

[8] Nieman, S. J. Schmetz, Johannes, Menzel, and W. Paul. A comparison of several techniques to assign heights to cloud tracers. Journal of Applied Meteorology, 32:1559-1568, 1993.

[9] J. Simpson and J. Gobat. Robust velocity estimates, stream functions, and simulated lagrangian drifters from sequential spacecraft data. IEEE trans. on Geosciences and Remote sensing, 32(3):479-492, 1994.

[10] D. Suter. Motion estimation and vector splines. In Proc. Conf. Comp. Vision Pattern Rec., pages 939-942, Seattle, USA, June 1994. 